\title{
Patrón de Conducta Tipo A y antecedentes familiares de hipertensión: un estudio psicofisiológico
}

\author{
E. Calvete Zumalde, L. de Nicolás y Martínez \\ Universidad de Deusto
}

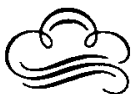

\section{Resumen}

En este estudio se evaluaron los efectos del Patrón de Conducta Tipo A y los antecedentes familiares de bipertensión en diversos parámetros cardiovasculares, conductuales y subjetivos de la respuesta de estrés.

Con este fin, 100 sujetos (50 varones y 50 mujeres) participaron en tareas de esfuerzo mental con control vs. no control. Las diferencias entre sujetos $A$ y $B$ se manifestaron en una mayor amplitud del pulso en los primeros. La mayor reactividad en Tasa Cardiaca se observó entre los sujetos con antece. dentes familiares de bipertensión, mientras que la presión sanguínea sistólica se vio condicionada por la interacción de dichos antecedentes con el Patrón de Conducta Tipo A. Finalmente, los componentes cardiovasculares, subjetivos y conductuales de la respuesta de estrés confirmaron la motivación por el control de dichos sujetos descrita en la literatura.

Palabras clave: Patrón de conducta tipo A, Hipertensión, Reactividad candiovascular, Estrés.

\section{Abstract}

The current study assessed the effects of the Type A Behavior Pattern and family bistory of hypertension on cardiovascular (pulse amplitude, beart rate and blood pressure), behavioral and subjective components of stress response.

With this aim 100 subjects (50 male and 50 female) participated in two control vs noncontrol experimental conditions. In comparison with Type B, Type A individuals showed a greater pulse amplitude in both conditions. Subjects who had a reported family history of bypertension bad a greater beart rate reactivity, while the sistolic blood pressure was affected by the interactionship between type A bebavior patterm and family history. Finally, cardiovascular, behavioral and subjetive components of stress response supported the bypothesis of control motivation.

Key words: Type A behavior pattern, Hypertension, Cardiovascular reactivity, Stress.

Dirección del autor: Dpto. de Personalidad, Evaluación y Tratamiento. Facultad de FICE.

Sección de Psicología. Universidad de Deusto. Apdo. 1, 48080 Bilbao. 


\section{INTRODUCCION}

Ninguna variable psicológica ha recibido tanta atención como el patrón de conducta tipo A en relación a la reactividad cardiovascular, hasta al punto que ha sido reconocido como importante factor de riesgo en el desarrollo de enfermedades coronarias.

Este síndrome conductual debe su nombre a los cardiólogos M. Friedman y R. Rosenman, quienes lo describieron como un conjunto de rasgos de hostilidad, agresividad, ambición, competitividad, alta implicación laboral e impaciencia crónica (Rosenman et al., 1975). En su nivel conductual, el conjunto se manifiesta por una tendencia a permanecer alerta y sin descanso, por un comportamiento precipitado, tensión en los músculos faciales y una forma de hablar explosiva. La antítesis es el patrón de conducta tipo $\mathrm{B}$, el cual se define por la relativa ausencia de las anteriores características.

En cuanto a los mecanismos que enlazan este patrón conductual con la enfermedad coronaria. La hipótesis más extendida consiste en la creencia de que los sujetos Tipo A exhiben una hiperreactividad cardiovascular y neuroendocrina que estimularía la formación de placas de arteriosclerosis (Lane et al., 1984). En esta línea, Muranaka et al. (1988) sugieren que los sujetos A y B difieren en su balance autonómico de modo que los primeros están predispuestos a una excesiva reactividad beta-adrenérgica y los segundos a respuestas alpha-adrenérgicas concurrentes con un mayor antagonismo parasimpático de los efectos beta-adrenérgicos.

Un aspecto importante es el referente a las dimensiones psicológicas claves que subyacen al patrón $A$ de conducta. Una de las líneas que más investigaciones ha promovido proviene del modelo de Glass (1977), según el cual las personas Tipo A están más motivadas a conseguir y mantener el control sobre los eventos ambientales y, en particular, sobre los eventos estresantes.

En consecuencia, el sujeto Tipo A se diferencia también en la forma de afrontar las situaciones estresantes, empleando más los métodos activos que las estrategias de evitación o pasivas (Smith y Brehm, 1981; Vingerhoets y Flohe, 1984; Calvete, 1989).

A partir de esta hipotetizada motivación por el control se han desarrollado numerosas investigaciones que, enlazando con la literatura sobre el afrontamiento activo-pasivo desarrollada fundamentalmente por el equipo de Obrist (Light y Obrist, 1983; Obrist et al., 1978), estudian la reactividad cardiovascular de los sujetos A y B bajo diferentes condiciones de control (véanse, por ejemplo, Contrada et al., 1982; Houston, 1986; Pittner y Houston, 1982; Pittner et al., 1983).

En cualquier caso, los numerosos estudios realizados en torno a la reactividad de estos individuos han dado lugar a resultados dispares, hasta el punto que algunos autores han propuesto el abandono del constructo (Linden, 1987).

Uno de los aspectos que puede dar lugar a la incongruencia de los resultados es el hecho de que el parámetro autonómico en el que se expresa la hiperresponsividad de los sujetos Tipo A depende de la naturaleza de la situación o tarea. En este sentido, Van Doornen (1988) sugiere que, a través de todas las tareas, el patrón $A$ es un hiperreactor en general, pero que el sistema en que dicha reactividad se expresa depende de la interacción entre patrón $\mathrm{A}$ y estresor. Cada estresor tiende a activar una determinada respuesta fisiológica. De este modo, dependiendo del estresor, las diferencias entre sujetos A y B se manifestarán en un patrón de respuesta u otro. 
Otro aspecto que ha podido condicionar la irregularidad de los resultados sobre los concomitantes fisiológicos del patrón de conducta Tipo A es su interacción con otros factores. En este sentido, diversos estudios (Katkin et al,, 1980; Williams et al., 1982; Lane et al., 1984) han encontrado una interacción entre el patrón de conducta Tipo A y los antecedentes familiares de enfermedad cardiovascular que puede predecir la magnitud de las respuestas cardiovasculares.

El papel de los antecedentes familiares de hipertensión aparece ampliamente documentado en la literatura, y parece asociarse a mayores elevaciones en Presión Sanguínea Sistólica durante una serie de breves estresores de laboratorio (Shapiro, 1961), mayores incrementos en Tasa Cardiaca y Presión Sanguínea Diastólica en tareas de evitación de descargas eléctricas (Jorgensen y Houston, 1981; Obrist et al., 1981), mayor Tasa Cardiaca y Presión Sanguínea Diastólica durante tareas aritméticas (Falkner et al., 1979; Jorgensen y Houston, 1981) y mayor Presión Sistólica en tareas de formación de conceptos, sustración serial y el «handgrip» (Manuck y Proietti, 1982).

A partir de los trabajos del equipo de Obrist, los antecedentes familiares de hipertensión se han considerado un factor determinante de la hiperreactividad cardiovascular como característica intrínseca de algunos sujetos (Light y Obrist, 1980; Obrist, 1987) y, consiguientemente, sus efectos deberían ser desligados de los de otros factores conductuales, tales como el Patrón de Conducta Tipo A.

En consecuencia, enlazando con estas ideas, el presente trabajo pretende profundizar en el estudio de los efectos del Patrón de Conducta Tipo A y de los antecedentes familiares de hipertensión en múltiples componentes fisiológicos, conductuales y subjetivos de la respuesta de estrés a condiciones experimentales de control vs. no control.

\section{METODO}

2.1. Sujetos. La muestra se compone de 100 estudiantes universitarios, de edades comprendidas entre los 19 y 37 años $(M=21.45$, DT $=4.21)$, de los cuales 50 son mujeres y 50 varones.

\subsection{Diseño.}

\subsubsection{Condiciones experimentales.}

Condición I: Prueba de dígitos asociada a ruido aversivo contingente a la ejecución.

El sujeto debe repetir series de números de dificultad máxima para él. Es decir, series de longitud igual $(n)$ o una mayor $(n+1)$ a la máxima que pudo repetir sin error durante la aplicación del Test de Dígitos del WAIS (Weschler, 1984).

Durante esta prueba, cada vez que el sujeto se equivoca comienza un ruido altamente aversivo (105 decibelios) que continúa hasta que éste es capaz de repetir otra serie correctamente.

Condición II: Prueba de dígitos combinada con ruido aversivo no contingente a la ejecución.

Esta prueba es similar a la anterior, salvo en el aspecto de la controlabilidad del ruido. En este caso el ruido comienza y termina de una manera preestableci- 


\section{8}

da, común a todos los individuos e independiente de los errores o aciertos en la ejecución.

\subsubsection{Variables, instrumentación y medición.}

Patrón de Conducta Tipo A/B y Antecedentes Familiares de Hipertensión.

Se ha empleado la Escala de Actividad de Jenkins (JAS), instrumento desarrollado para medir la conducta tipo $\mathrm{A}$ de forma más rápida y económica que a través de la entrevista estructurada (Jenkins et alt., 1979).

En este estudio se ha empleado la versión para población española de De Flores, Valdés y Sans (1982). La corrección de los resultados ha sido realizada por el Dr. Tomás de Flores del Servicio de Psicología Médica de la Facultad de Medicina de Barcelona.

A efectos del análisis se trabajó con los sujetos de percentil menor o igual que 30 (Tipo B) y mayor o igual que 70 (Tipo A).

Asimismo, se obtuvo información referente a la presencia de hipertensión en los padres de los sujetos participantes.

\section{Variables Fisiológicas.}

Se registraron la Tasa Cardiaca (latidos por minuto), Amplitud del Pulso Sanguíneo (MV), Tasa Respiratoria y Presión Sanguínea Sistólica y Diastólica $(\mathrm{Hg}$ $\mathrm{mm})$.

Todas las variables fisiológicas, excepto la presión sanguínea, fueron registradas por un Polígrafo Grass modelo 7D equipado con amplificadores DC modelo 7P122. Para la medición de la amplitud del pulso y tasa cardiaca se empleó un pletismógrafo Grass modelo PTTL, colocado en el lóbulo de la oreja derecha. La tasa respiratoria fue obtenida mediante un trasductor de la temperatura Grass modelo TCT 1R. Esta variable fue utilizada con el único fin de verificar la ausencia de maniobras respiratorias peculiares (por ejemplo, respiración sostenida) como respuesta consistente a las tareas (Jennings y Choi, 1981).

La presión sanguínea se obtuvo mediante un medidor digital modelo DS-91, procediéndose a su anotación mecánica. Todas las demás señales fueron digitalizadas por un convertidor analógico-digital DT2814 a una tasa de 30 muestras por segundo y grabadas en un ordenador personal IBM en la siguiente secuencia de seis ensayos: último minuto de la Línea de Base para cada condición experimental y primer y cuarto minutos de las Condiciones I y II.

La grabación y análisis de estos ensayos para la obtención de los niveles promedios en amplitud del pulso (MV), tasa cardiaca y tasa respiratoria fueron realizados mediante el software cedido amablemente por el Dr. Jaime Vila de la Universidad de Granada.

\section{Variables Subjetivas.}

Mediante los cuestionarios ESE (Experiencia Subjetiva de Estrés) y EAS (Evaluación y Afrontamiento de la Situación) (Calvete, 1990) se evaluaron los siguientes factores subjetivos del estrés y afrontamiento de las situaciones: Experiencia de Estrés, Activación Positiva, Activación Negativa, Enojo, Autocrítica de la Ejecución, Afrontamiento Activo, Afrontamiento Pasivo y Fatalismo, Evaluación Negativa de la Situación. 
Variables Conductuales

Se midió el nivel de ejecución, operativizado en el número de aciertos en la repetición de dígitos en sentido directo e inverso para cada condición experimental.

\subsection{Procedimiento.}

Las citas en el laboratorio fueron concertadas telefónicamente. Se pidió a los sujetos que se abstuviesen de consumir café, alcohol y tabaco dos horas antes del experimento.

Tras un período de familiarización, se colocaban los transductores, se aplicaba el test de dígitos y se dejaban transcurrir veinte minutos de adaptación. $\mathrm{Al}$ comienzo de los mismos, el sujeto contestaba el cuestionario ESE a fin de poder evaluar su estado subjetivo antes de los experimentos. Se medía, también, su presión sistólica y diastólica.

A continuación, se presentaban las condiciones experimentales - precedidas cada una por una breve descripción - en dos órdenes diferentes: 50 individuos ( 25 mujeres y 25 varones) las realizaron en el orden I-II mientras que al resto, se les presentaron en el orden II-I. De esta manera se neutralizaron posibles interacciones entre las dos condiciones de dígitos más ruido.

Inmediatamente después de cada condición experimental, se procedía a medir la presión sanguínea del individuo y, a continuación, el sujeto contestaba a los cuestionarios ESE y EAS, refiriéndose a lo que había experimentado durante la prueba. Tras la cumplimentación de las escalas, se dejaba transcurrir un período de adaptación de diez minutos a fin de que las diversas variables fisiológicas recuperasen los niveles basales.

\section{Resultados.}

\subsection{Diferencias en reactividad según la Escala $A / B$.}

\section{Tasa cardiaca.}

En la Tabla I se recogen las medias y desviaciones típicas para la Tasa Cardiaca y Amplitud del Pulso durante las dos condiciones experimentales, para los sujetos A y B.

\section{TABLA I}

Medias $y$ desviaciones típicas en amplitud del pulso y tasa cardiaca para los sujetos Tipo A y Tipo B.

CONTROL

$\begin{array}{rrrr}\mathrm{AP}, 1 .^{\mathrm{er}} \mathrm{min} . & 2.03(1.91) & 2.40(1.69) \\ 4 .^{\circ} \mathrm{min} . & 2.09(1.26) & 2.32(1.51) \\ \mathrm{TC}, 1 .^{\mathrm{er}} \mathrm{min} . & 85.56(10.09) & 86.80(10.55) \\ 4 .^{\circ} \mathrm{min} . & 80.54(7.90) & 81.25(8.10)\end{array}$

NO CONTROL

$\begin{array}{rrrrr}\mathrm{AP}, 1 .^{\mathrm{er}} \mathrm{min} . & 2.21 & (1.55) & 2.53 & (1.72) \\ 4 .^{\circ} \mathrm{min} . & 2.10 & (1.38) & 2.62 & (1.74) \\ \mathrm{TC}, 1 .^{\mathrm{er}} \mathrm{min} . & 82.79 & (8.19) & 81.98 & (8.25) \\ 4 .^{\circ} \mathrm{min} . & 79.29 & (7.05) & 80.29 & (7.94)\end{array}$


No se han encontrado diferencias en tasa cardiaca entre sujetos A y B para ninguna de las condiciones experimentales.

Amplitud del pulso.

Un MANCOVA de medidas repetidas con diseño 2 (control, no control) $\times 2$ (primer y cuarto minutos) $\times 2$ (Patrón $A / B)$ y como covariante la amplitud del pulso durante las líneas bases señala la significación del efecto del Patrón $A\left[F_{1,57}=6.12, p<0.01\right]$. Sin embargo, no se observa un efecto significativo de la interacción entre el patrón de conducta con la condición experimental ni con el tiempo. Lo que sí aparece como marginalmente significativo, es el efecto de la interacción entre dicho patrón y la condición y el tiempo simultáneamente $\left[F_{1,57}=3.08, p<0.08\right]$. Dicha interacción se muestra en la Figura 1 y puede describirse como sigue: En la situación de control, los sujetos A experimentan una mayor vasoconstricción con el paso del tiempo, mientras que los sujetos B se estabilizan. En la situación de no control, en cambio, los sujetos $\mathrm{A}$ se habitúan algo, mientras que los $\mathrm{B}$ experimentan una mayor vasoconstricción.

\section{FIGURA I}

Diferencias en pulso entre sujetos $A$ y $B$ en condiciones de control y no control

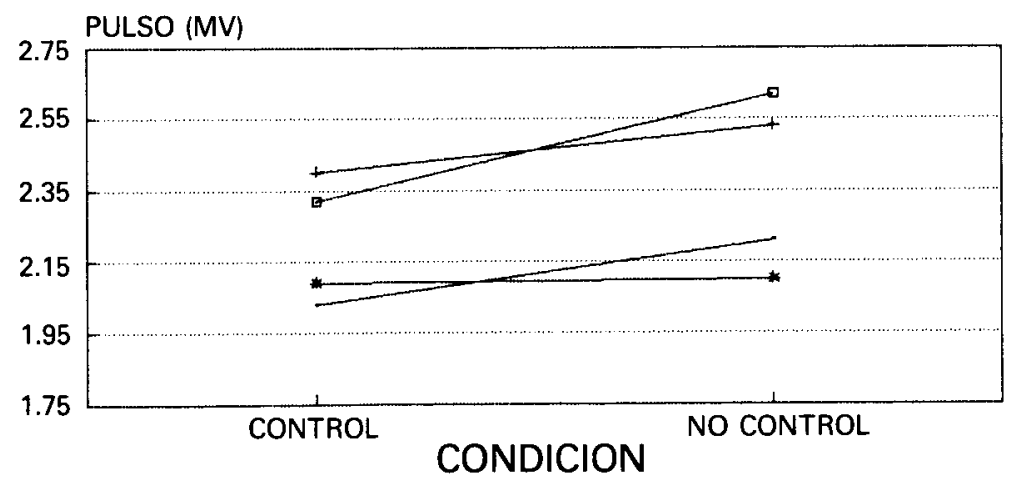

- B PRIMER MI — A PRIMER MI * B CUARTO MI $\rightarrow$ A CUARTO MI

\section{Presión sanguínea.}

Aunque se observan mayores presiones en los sujetos Tipo A durante todas las condiciones, las diferencias no son significativas.

\section{Variables subjetivas.}

En la condición I (control), los sujetos A se sienten más activados, tal y como se deduce de una mayor puntuación en el factor de «activación positiva» $\left[\mathrm{M}=15.95(3.00)\right.$ vs. $\left.\mathrm{M}=17.70(3.82), \mathrm{F}_{1,44}=2.96, \mathrm{p}<0.09\right]$ y menor en el factor de «activación negativa» $[M=4.27(1.64)$ vs. $M=3.08(2.71)$, $\left.\mathrm{F}_{1,44}=3.16, \mathrm{p}<0.08\right]$. Además, la autocrítica de la situación resulta menor que en el caso de los sujetos Tipo $B[M=7.22$ (4.11) vs. $M=5.33$ (3.29), $\left.\mathrm{F}_{1,44}=2.99, \mathrm{p}<0.09\right]$. 
Durante la condición II (no control), los sujetos Tipo A experimentan un mayor grado de estrés que los Tipo $B[M=17.15$ (6.97) vs. $M=20.28$ (6.69), $\left.\mathrm{F}_{1,52}=2.83, \mathrm{p}<0.09\right]$.

\section{Ejecución.}

$\mathrm{El}$ análisis de la varianza de diseño 2 (condición I/II) $\times 2$ (Tipo A/B) señala un efecto significativo de la interacción entre el patrón de conducta Tipo A y la condición sobre el número de aciertos en la repetición de dígitos en orden directo $\left[\mathrm{F}_{1,60}=6.33, \mathrm{p}<0.01\right]$. Mientras que, en la condición de control, los sujetos de alta implicación laboral obtienen mejores resultados en su rendimiento $[M=5.19$ (2.01) vs. $M=6.2(1.9)]$; en la condición de no control el efecto se invierte $[M=5.56(1.92)$ vs. $M=5.33(2.04)]$.

\subsection{Antecedentes Familiares de Hipertensión y Patrón de Conducta Tipo A.}

\section{Tasa cardiaca.}

Se ha realizado un Análisis de la Varianza de diseño 2 (A, B) $\times 2$ (con antecedentes, sin antecedentes) $\times 2$ (tasa cardiaca en las dos condiciones experimentales) y con los valores basales como covariante. El resultado no muestra un efecto significativo de la interacción de ambos factores, pero sí de los antecedentes familiares de hipertensión.

\section{TABLA II}

Diferencias en tasa cardiaca en función de los antecedentes familiares de bipertensión.

\begin{tabular}{lcccc}
\hline & Sin antecedentes & Con antecedentes & $\mathrm{F}_{1,63}$ & Signif. \\
\hline Control & $77.58(8.62)$ & $86.13(11.94)$ & 11.27 & 0.001 \\
No control & $76.88(10.16)$ & $82.53(10.74)$ & 4.65 & 0.03 \\
\hline
\end{tabular}

Los hijos de personas con hipertensión obtienen valores más altos en tasa cardiaca en las dos condiciones experimentales, tal y como se recoge en la Tabla II.

Amplitud del pulso.

Tampoco se observa un efecto significativo para la interacción entre Patrón de Conducta y Antecedentes Familiares sobre la AP. Sí se observan efectos de los antecedentes solos en los cambios de AP durante la condición de control. Los sujetos con padres hipertensos presentan una mayor vasoconstricción durante esta condición, tanto durante el primer minuto $[\mathrm{M}=-0.87(0.75)$ vs. $\left.\mathrm{M}=-0.44(0.74), \mathrm{F}_{1,63}=5.31, \mathrm{p}<0.02\right]$ como durante el cuarto $[\mathrm{M}=$ $-0.78(0.89)$ vs. $\left.\mathrm{M}=-0.31(0.79), \mathrm{F}_{1,63}=4.91, \mathrm{p}<0.03\right]$.

\section{Presión sanguinea.}

No se han encontrado diferencias en tensión sanguínea entre los sujetos con dichos antecedentes y los que no los tenían. Sin embargo, se observa un 
efecto significativo de la interacción de esta variable con el Patrón de Conducta en la PSS correspondiente a las condiciones de control $\left[F_{1,40}=3.54\right.$, $p<0.06]$ y no control $\left[F_{1,39}=6.03, p<0.02\right]$, que se explica por una mayor PSS entre los sujetos Tipo A con antecedentes, mientras que, de los Tipo B, son aquellos sin antecedentes los que alcanzan mayores valores (Tabla II).

\section{DISCUSION}

Los resultados de este estudio no han arrojado diferencias en tasa cardiaca entre sujetos Tipo A y Tipo B y aunque los primeros presentaron mayores valores en presión sanguínea sistólica y diastólica en las dos condiciones experimentales, las diferencias no fueron estadísticamente significativas. Estos parámetros fisiológicos parecen estar condicionados por los antecedentes familiares de hipertensión. De esta manera, los sujetos que presentaban dichos antecedentes exhibieron una mayor reactividad en tasa cardiaca tanto en la condición de control como de no control. Combinando el Patrón de Conducta Tipo A/B con los historia familiar, se ha encontrado una mayor presión sistólica en los sujetos Tipo A con antecedentes familiares de hipertensión, al igual que encontraron Lane et al. en 1984.

Con todo, los datos más relevantes se han obtenido para la Amplitud del $P u l s o$, delineándose un patrón diferencial de reactividad en los sujetos $\mathrm{A} / \mathrm{B}$ en función de la situación:

Durante las dos condiciones de dígitos la resistencia periférica es mayor en los sujetos Tipo B. A esto hay que añadir un efecto diferencial curioso en relación al paso del tiempo: los sujetos Tipo A experimentan una pequeña sensibilización de la respuesta en la situación de no control y una pequeña habituación en la de control, mientras que se observan los efectos contrarios en los sujetos Tipo B.

Los resultados referentes a la amplitud del pulso requieren una consideración especial, puesto que, como Blumenthal et al. (1985) sugirieron, las medidas de actividad vasomotora podrían ser el discriminador más sensitivo de la hiperresponsividad cardiovascular que caracteriza a los sujetos Tipo A.

Con anterioridad, Scherwitz et al. (1978) habían encontrado en los sujetos Tipo A una mayor amplitud del pulso en el dedo durante tareas de aritmética, «Cold Pressor Test», Entrevista Estructurada y Expresión de Emociones. Van Egeren (1979) obtuvo una correlación de .45 entre la amplitud del pulso digital y el Patrón de Conducta Tipo A durante el período anticipatorio de una condición competitiva.

Otros estudios han encontrado mayor vasodilatación entre los sujetos $\mathrm{A}$ en los músculos esqueléticos del brazo, en situaciones de aritmética mental (Blumenthal et al., 1985; Lane et al., 1984; Williams et al., 1982). Todos estos indicios reclaman una mayor atención futura para la actividad vasomotora en el estudio de la relación entre estrés y Patrón de Conducta Tipo A, línea de investigación que tradicionalmente se ha centrado en las medidas de presión sanguínea y tasa cardiaca.

Con respecto a los componentes subjetivos de la respuesta de estrés y afrontamiento, encontramos que los sujetos Tipo A, en comparación a los B, manifiestan sentirse más estresados en la condición de no control, mientras que en la de control lo que expresan es una mayor activación y satisfacción con su ac- 
tuación. Esto es coherente con los cambios mencionados en la amplitud del pulso.

Las diferencias en variables conductuales se han observado en relación a la controlabilidad de la situación. Los sujetos Tipo A presentan una mejor ejecución en la condición de control que los Tipo $\mathrm{B}$, mientras que el efecto se invierte para la condición de no control.

Por tanto, la mayor habituación de la respuesta vasomotora, la mejor ejecución de dicha tarea y la percepción de la misma como de menor poder estresante confirman la motivación por el control de los sujetos Tipo A, tal y como se alude en la literatura.

Concluyendo, este estudio no ha encontrado diferencias significativas entre sujetos Tipo A y B en los parámetros de Tasa Cardiaca y Presión Sanguínea para tareas de esfuerzo mental con distinto grado de control. En contraste, ambos tipos de individuos han exhibido patrones diferenciales de reactividad en amplitud del pulso sanguíneo, correspondiendo una respuesta vasodilatadora a los sujetos Tipo A. La motivación por el control descrita en la literatura se ha manifestado tanto en los componentes fisiológicos como subjetivos y conductuales de la respuesta de estrés. Finalmente, las diferencias en tasa cardiaca han estado determinadas por los antecedentes familiares de hipertensión, mientras que las diferencias en presión sanguínea sistólica se han debido a la interacción de dichos antecedentes con el patrón de conducta Tipo A.

\section{Referencias}

Blumenthal, J. A.; Lane, J. D. y Williams, R. B. (1985): The inhibited power motive, type A behavior, and patterns of cardiovascular response during the Structured Interview and Thematic Apperception Test. Journal of Human Stress, 82-92.

Calvete, E. (1989): Factores disposicionales del afrontamiento al estrés diario: Patrón de conducta Tipo A y Locus de Control. Revista Vasca de Psicología, 2 (1), 5-17.

Calvete, E. (1990): Componentes cardiovasculares y subjetivos del proceso de estrés: Factores psicosociales. Universidad de Deusto. Bilbao.

Contrada, R. J.; Glass, D. C.; Krakoff, L. R.; Krantz, D. S. (1982): Effects of Control Over Aversive Stimulation and Type A Behavior on Cardiovascular and Plasma Catecholamine Responses. Psychopbysiology, 19 (4), 408-419.

Contrada, R. J. et al. (1984): Task difficulty, type A pattern and cardiovascular response. Psycbopbysiology, 21, 638-646.

De Flores, T., Valdếs, M. y Sans, S. (1982): Inventario de Actividad de Jenkins. Edición experimental. Madrid: TEA.

DE NICOLÁs, L. (1988): El estrés en la sociedad actual: Modelos terapéuticos. Cuadernos de Extensión Universitaria. Universidad del País Vasco. Bilbao.

De Nicolás, L. y Matute, H. (1984): Control congnitivo de reacciones emocionales ante una reacción estresante. Letras de Deusto. Univ. de Deusto. Bilbao.

FaLKNER, B. et al. (1979): Cardiovascular response to mental stress in normal adolescents with hypertensive parents. Hypertension, 1, 23-30.

Glass, D. C. (1977): Behavior patterns, stress and coronary disease. Hillsdale: John Wiley y Sons.

Lane, J. D., White, A. D. y Williams, R. B. Jr. (1984): Cardiovascular effects of mental arithmetic in type A and type B females. Psychopbysiology, 21 (1), 39-46.

Jenkins, C. D.; Zyzansky, S. J. y Rosenman, R. H. (1979): Jenkins Activity Survey Manual. Nueva York: The Psychological Corporation.

Jennings, J. R. y Chol, S. (1981): Type A components and psychophysiological responses to an attention-demanding performance task. Psychosomatic Medicine, 43 (6), 475-487.

Jorgensen, R. S. y Houston, B. K. (1982): The type A behavior pattern, sex differences, and cardiovascular response to and recovery from stress. Motivation and Emotion, 5 , 201-214. 
Katkin, E. S.; Goldband, S. y Medin, B. (1980): Cardiovascular responses of type A and type B subjects differing in family history of heart disease (Abstract). Psychophysiology, $17,319$.

Light, K. C. у ObRist, P. A. (1983): Task difficult, Heart Rate Reactivity, and Cardiovascular Responses to an appetitive reaction time task. Psychophysiology, 20 (3), 301-312.

Manuck, S. B. y ProletTI, J. M. (1982): Parental Hypertension and Cardiovascular Response to Cognitive and Isometric Challenge. Psychopbysiology, 19 (5), 481-489.

MuRANAKA, M. et al. (1988): Stimulus-specific patterns of cardiovascular reactivity in type $A$ and $B$ subjects: Evidence for enhanced vagal reactivity in type B. Psychophysiology, 25 (3), 330-338.

OBRIST, P. A. (1985): Beta-adrenergic hyperresponsivity to behavioral challenges: a possible hypertensive risk factor. En J. F. Orlebeke et al. (Eds.), Psychophysiology of cardiovascular control. Nueva York: Plenun Press.

Obrist, P. A.; Gaebelein, C. J.; Teller, E. S.; Langer, A. W.; Grignolo, A., et al. (1978): The Realtionship Among Heart Rate, Carotid $\mathrm{dP} / \mathrm{dt}$, and Blood Pressure in Humans as a Function of the Type of Stress. Psychophysiology, 15 (2), 102-115.

OBRIST, P. A.; LIGHT, K. A., et al. (1981): Beta-adrenergic hyperreactivity and behavioral stress as precursor to established essential hypertension? En S. Weiss et al. (Eds.), Perspectives in behavioral medicine. Nueva York: Academic Press.

Obrist, P. A.; Light, K. C.; James, S. A. y Strogatz, D. S. (1987): Cardiovascular responses to stress: I. Measures of Myocardial Response and relationship to hight resting systolic pressure and parental hypertension. Psychophysiology, 24 (1), 65-78.

PitTner, M. S. y Houston, B. K. (1980): Response to stress, cognitive coping strategies, and the type A behavior pattern. Joumal of Personality and Social Psychology, 39, 147-157.

PitTNer, M. S.; Houston, B. K. y Spiridigliozzi, G. (1983): Control over stress, type A behavior pattern, and response to stress. Joumal of Personality and Social Psychology, 44 (3), 627.637.

Rosenman, R. H.; Brand, R. J.; Jenkins, C. D.; Friedman, M.; Straus, R.; Wurm, M. (1975): Coronary heart disease in the Western Collaborative Group Study: Final follow-up experience of $8^{1 / 2}$ years. Joumal of the American Medical Association, 233.

ShAPIRO, A. P. (1961): An experimental study of comparative responses of blood presure to different noxious stimulus. Journal of Chronic Diseases, 13, 293-311.

ScHERWITz, L. et al. (1978): Type A behavior, self-involvement, and cardiovascular response. Psychosomatic Medicine, 40 (8), 593-609.

SMITH, T. W. y BREHM, S. S. (1981): Cognitives correlates of the type A Coronary-prone behavior pattern. Motivation and Emotion, 215-223.

Vingerhoets, A. J. J. M. y Flohr, P. J. M. (1984): Type A behavior and self-reports of coping preferences. British Journal of Medical Psychology, 57, 15-21.

VAN DOORNEN, L. J. P. (1988): Physiological stress reactivity: Its realtionship to behavioral style, mood, sex, and aerobic fitness. Doctoral Dissertation. Vrije Universiteit te Amsterdam.

VAN EGEREN, L. F. (1979): Social Interactions, communications, and the coronary-prone behavior pattern: A psychophysiological study. Psychosomatic Medicine, 41 (1), 2-18.

Williams, R. B. et al. (1982): Type A behavior and elevated physiological and neuroendocrine responses to cognitive tasks. Science, $218,483.485$. 